\title{
Rupture of Membrane
}

National Cancer Institute

\section{Source}

National Cancer Institute. Rupture of Membrane. NCI Thesaurus. Code C92874.

Breaking or tearing of the amniotic membranes during childbirth leading to the leakage of amniotic fluid and the progression of labor. 